\title{
Chiral Biphenyldicarboxylic Acid Networks Stabilized by Hydrogen Bonding
}

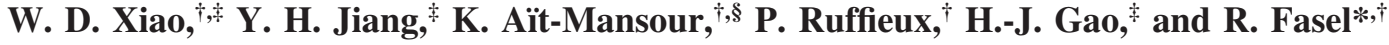 \\ Empa, Swiss Federal Laboratories for Materials Testing and Research, Feuerwerkerstrasse 39, \\ CH-3602 Thun, Switzerland, Institute of Physics, Chinese Academy of Sciences, 100190 Beijing, China, and \\ IPMC, Ecole Polytechnique Fédérale de Lausanne, Station 3, CH-1015 Lausanne, Switzerland
}

Received: January 25, 2010

\begin{abstract}
The self-assembly of 4,4'-biphenyldicarboxylic acid on a $\mathrm{Au}(111)$ surface is investigated by means of in situ ultra-high-vacuum scanning tunneling microscopy. Three types of open networks with increasing interpore distance are observed. Experiments and theoretical calculations reveal that the two-dimensional porous networks are stabilized by various types of hydrogen bonds between carboxyl groups and by van der Waals interactions. Cyclic tetrameric and half-cyclic trimeric hydrogen-bonding motifs are shown to induce chirality in the open networks.
\end{abstract}

\section{Introduction}

The self-assembly of organic molecular building blocks on surfaces is a promising route toward functional nanoarchitectures. ${ }^{1}$ Among the great variety of molecular building blocks, molecules exhibiting carboxyl groups are particularly prominent examples ${ }^{2}$ because of the capability of the carboxyl groups to form robust hydrogen bonds (HBs) with the hydroxyl subgroup acting as a donor and the carbonyl oxygen as an acceptor. ${ }^{2}$ Furthermore, carboxyl groups can deprotonate into carboxylates, which are prone to coordinate with metal atoms. ${ }^{3}$ To date, the most common H-bonding motifs for carboxylic acids both on surfaces and in three-dimensional (3D) molecular crystals are cyclic dimers, cyclic trimers, and catemeric motifs., ${ }^{2,4}$

The 4,4'-biphenyldicarboxylic acid (BPDA) molecule consists of two phenyl rings and two carboxyl end groups (see the inset of Figure 1a) and has been successfully exploited for the fabrication of highly organized assemblies at surfaces. ${ }^{2,5-8}$ Stepanow and co-workers reported that, upon adsorption on the $\mathrm{Cu}(100)$ surface at room temperature (RT), the BPDA molecules form densely packed and ordered molecular adlayers with fully deprotonated carboxylate moieties via lateral HBs between the carboxylato oxygens and the phenyl groups of the adjacent molecules, resulting in a perpendicular configuration of each molecule to its four nearest neighbors. ${ }^{5}$ Addition of iron atoms to the BPDA adlayer leads to the formation of metal-organic coordination networks with rectangular pores, where the carboxyl groups are also deprotonated. ${ }^{6,7}$ Recently, Zhu et al. observed that BPDA molecules are connected through linear 2-fold HBs with carboxyl groups of adjacent molecules and form ordered close-packed islands on the $\mathrm{Au}(111)$ surface, where the molecules in the same islands adopt a parallel configuration. ${ }^{8}$ The different behavior of BPDA molecules self-organization on $\mathrm{Cu}(100)$ and $\mathrm{Au}(111)$ surfaces was attributed to the presence (absence) of deprotonation of the carboxyl groups. ${ }^{8}$

Herein, we report on the formation of chiral porous networks, as well as close-packed islands, with $C_{2}$-symmetric BPDA molecules on $\mathrm{Au}(111)$. Although the BPDA molecules are linear

* To whom correspondence should be addressed. E-mail: roman.fasel@ empa.ch.

${ }^{\dagger}$ Empa, Swiss Federal Laboratories for Materials Testing and Research.

* Institute of Physics, Chinese Academy of Sciences.

${ }^{\S}$ Ecole Polytechnique Fédérale de Lausanne. and each includes two carboxyl end groups, in situ ultra-highvacuum scanning tunneling microscopy (STM) investigations and theoretical calculations show that the BPDA molecules selfassemble into a series of two-dimensional (2D) chiral porous networks via various H-bonding motifs with carboxyl groups, highlighting the flexibility of carboxylic acids in the synthesis of surface-supported nanoarchitectures. ${ }^{2}$

\section{Experiments}

Experiments were performed in an ultra-high-vacuum variable temperature STM system (Omicron) equipped with standard surface preparation facilities. The Au(111) surface was prepared by repeated cycles of sputtering with argon ions and annealing at $450{ }^{\circ} \mathrm{C}$. The BPDA molecules (Aldrich, 97\% purity) were deposited via vacuum sublimation from a Knudsen-type evaporator. One monolayer (ML) refers to the completion of a closepacked BPDA layer on $\mathrm{Au}(111)$, as estimated with STM. STM images were acquired in constant-current mode at a sample temperature of $\sim 50 \mathrm{~K}$.

Molecular modeling was performed with the HyperChem, version 7 , program. ${ }^{9}$ Optimization in vacuum at the AM1 level of theory gives two (meta)stable molecular configurations: a fully planar one and an energetically slightly more favorable configuration with the two phenyl rings twisted by about $47^{\circ}$. Because the molecule-substrate interaction overrules the weak steric repulsion between the phenyl ring hydrogens, BPDA molecules adopt a planar configuration after adsorption on $\mathrm{Au}(111)$. Therefore, we use the planar conformation after optimization in vacuum at the PM3 level, which gives a molecular configuration with $C_{2}$ symmetry.

Intermolecular interactions were investigated by total energy calculations performed using the Amber3 force field, as implemented in the HyperChem, version 7, program. ${ }^{9}$ The carboxyl groups were fully optimized, while the other atoms in the molecules were fixed in these calculations. Interaction energies were calculated for different intermolecular distances and relative molecular orientations. The effect of the surface was accounted for by forcing molecules to lie within the same plane. To cross-check the configurations obtained by Amber3 force field calculations, density functional theory (DFT) calculations were performed at the X3LYP/6-31G(d) level using the PC GAMESS, version 7, program. ${ }^{10,11}$ 

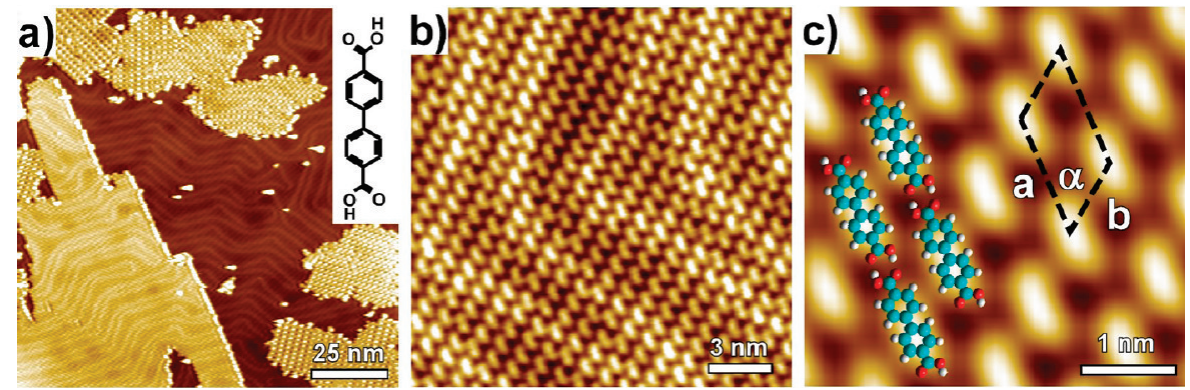

Figure 1. (a) Overview STM image showing the coexistence of open networks and close-packed islands of BPDA molecules. The inset shows the chemical structure of BPDA. (b) Large-scale STM image showing the long-range ordered close-packed islands. (c) Close-up of the close-packed islands. The unit cell and structural model are superposed.

\section{Results and Discussion}

After vapor deposition of $\sim 0.3 \mathrm{ML}$ of BPDA molecules on an atomically clean $\mathrm{Au}(111)$ surface at RT, we observe by STM a variety of ordered porous phases as well as densely packed islands at a sample temperature of $\sim 50 \mathrm{~K}$, as seen in Figure 1a. Large-scale STM images (Figure 1b) demonstrate the formation of long-range ordered close-packed islands of the BPDA molecules on the Au(111) surface. A zigzag pattern can be clearly seen as a modulation of the extended islands contrast in the STM image. This zigzag pattern stems from the $\mathrm{Au}(111)$ $22 \times \sqrt{ } 3$ herringbone reconstruction, ${ }^{12}$ which evidences that the $\mathrm{Au}(111)$ surface reconstruction is not destroyed or lifted upon deposition of the BPDA molecules, suggesting a weak molecule-substrate interaction. Images at high magnification, as the one shown in Figure 1c, illustrate a regular lattice with lattice parameters of $a=1.29 \pm 0.06 \mathrm{~nm}, b=0.75 \pm 0.05$ $\mathrm{nm}$, and $\alpha=50^{\circ} \pm 2^{\circ}$. Each molecule shows a rod feature, which originates from the aromatic backbones. Although the phenyl rings of BPDA molecules are twisted in vacuum, the molecules adopt a flat adsorption configuration because of the $\pi-$ metal interaction. Amber3 force field and DFT calculations (see the Supporting Information) unveil that the BPDA molecules in the close-packed islands are connected head-to-tail via 2-fold cyclic HBs between carboxyl groups of adjacent molecules, forming one-dimensional (1D) linear molecular chains, which further close-pack into 2D extended ordered islands via van der Waals ( $\mathrm{vdW}$ ) interactions. The corresponding structural model is superposed to Figure 1c. This cyclic dimeric HB pattern can be described as $R_{2}^{2}(8)$ in the graph-set notation of Etter and co-workers. ${ }^{13}$ Similar self-assembly was also observed for various aromatic linear dicarboxylic acids on metal or graphite surfaces. ${ }^{8,14}$

Of particular interest is the formation of porous networks. The overview STM image in Figure 2a illustrates the mesoscopic ordering of BPDA porous networks in various domains. Highly magnified STM images (Figure $2 b-e$ ) reveal the coexistence of three ordered phases, which we denote as A, B, and C. These ordered phases display similar networks with square pores, although the detailed molecular arrangement and coordination are different. The A and B phases can form extended domains, whereas the C-phase domains are much smaller and appear considerable less frequently.

The A phase demonstrates a square lattice with a lattice parameter (pore-to-pore distance) of $a=1.66 \pm 0.05 \mathrm{~nm}$. Each unit cell consists of two molecules that are perpendicular to each other. The basic bonding configuration of the BPDA molecules in the A phase is a distorted cross that includes four BPDA molecules, as seen in Figure 2b,c. Amber3 force field and DFT calculations (see the Supporting Information) reveal
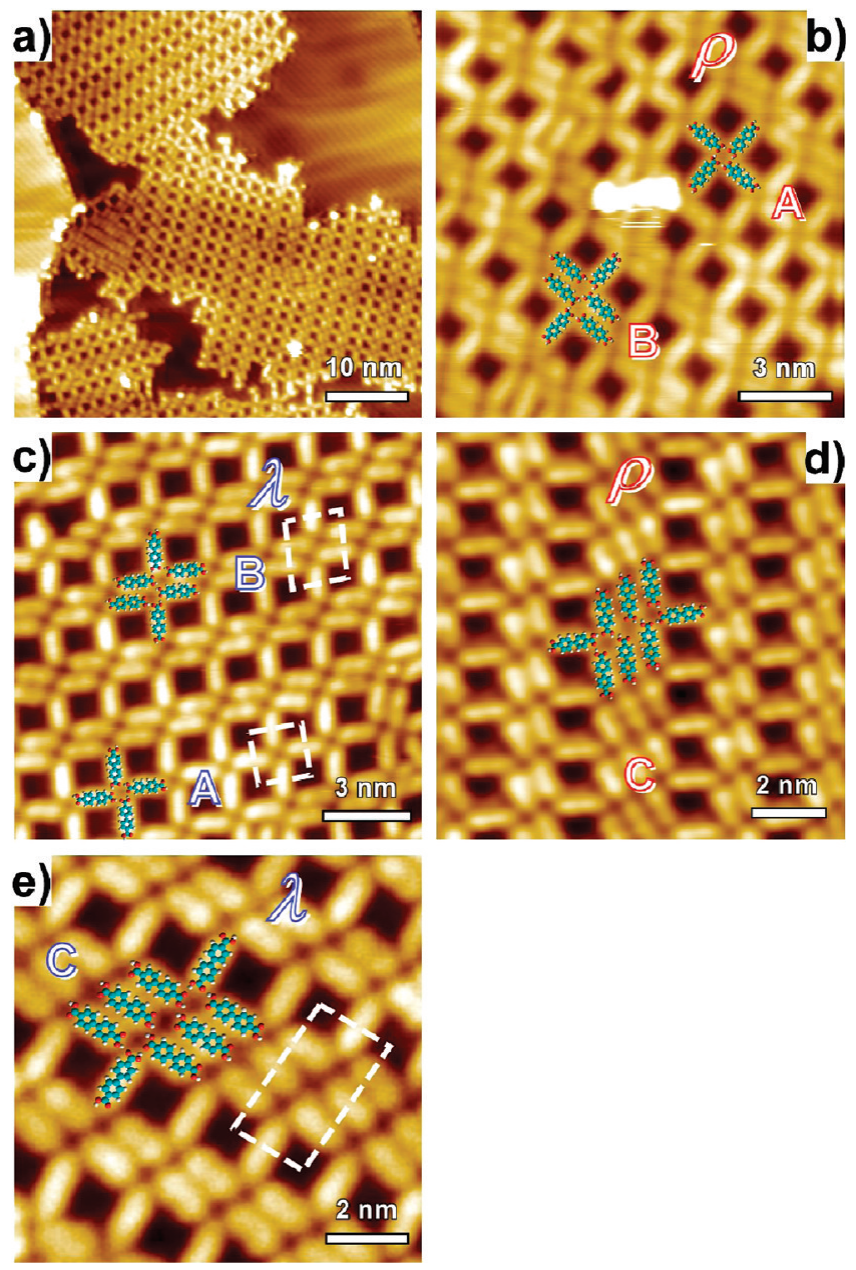

Figure 2. STM images of ordered chiral open networks after deposition of $0.3 \mathrm{ML}$ of BPDA molecules on $\mathrm{Au}(111)$ at room temperature. (a) Large-scale image showing the mesoscopic ordering of BPDA porous networks in various domains. (b) $\mathrm{A}$ and $\mathrm{B}$ phases of the $\rho$ domain. (c) $\mathrm{A}$ and $\mathrm{B}$ phases of the $\lambda$ domain. (d) $\mathrm{C}$ phase of the $\rho$ domain. (e) $\mathrm{C}$ phase of the $\lambda$ domain. The unit cells of the A, B, and $\mathrm{C}$ phases are marked with white dashed lines, and the corresponding structural models are superposed.

that the four molecules are connected via cyclic HBs between carboxyl groups, corresponding to a graph-set notation of $R_{4}{ }^{4}(16) \cdot{ }^{13}$ The calculated intermolecular separation of $1.66 \mathrm{~nm}$ between two parallel molecules is well-consistent with STM measurements. We note that this unique cyclic tetrameric HB pattern was rarely reported and, ${ }^{15}$ in particular, never observed so far in the self-assembly of aromatic linear dicarboxylic acids on surfaces. Interestingly, both STM experiments and theoretical calculations reveal that the long axes of BPDA molecules are 


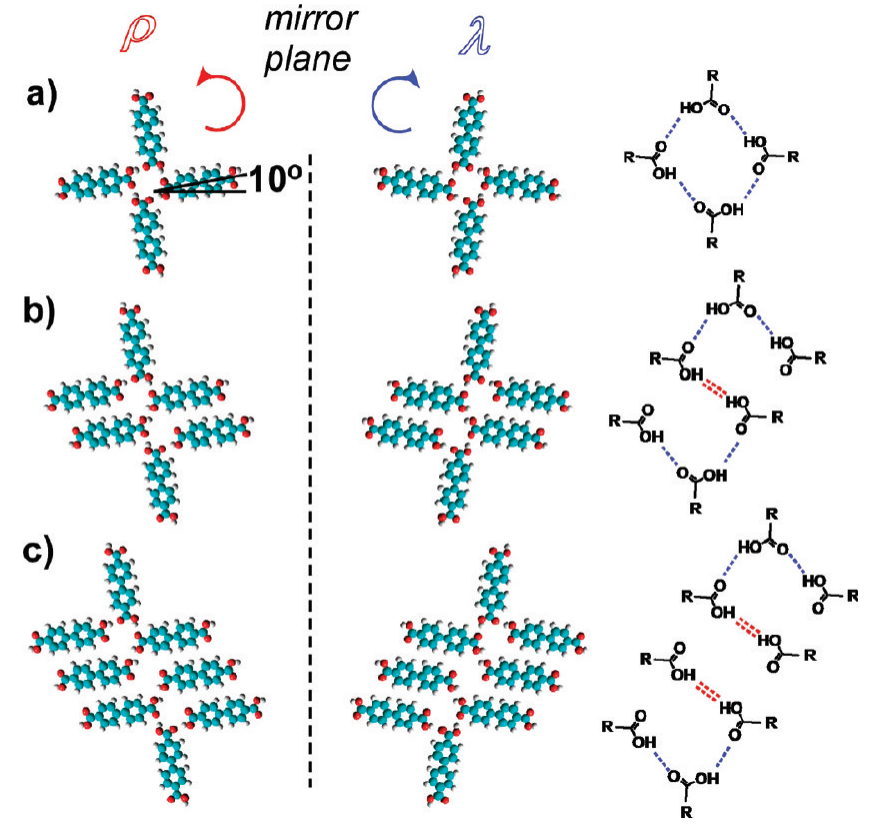

Figure 3. Structural models of the A, B, and C phases of the $\lambda$ and $\rho$ chiral domains and their basic bonding configurations. (a) A phase. (b) B phase. (c) C phase. Blue single and red double dashed lines illustrate two different types of HBs.

rotated away from the lattice vectors of the molecular network by $\sim 10^{\circ}$, inducing $2 \mathrm{D}$ chirality into the BPDA networks (Figure $2 \mathrm{~b}, \mathrm{c})$. Structural models of the left- and right-handed $\lambda$ and $\rho$ domains and the connection of the cross of the A phase via cyclic HBs are illustrated in Figure 3a.

Different from the square lattice of the A phase, the B phase demonstrates an oblique lattice with lattice parameters of $a=$ $1.67 \pm 0.05 \mathrm{~nm}, b=2.03 \pm 0.05 \mathrm{~nm}$, and $\alpha=82 \pm 2^{\circ}$, as seen in Figure 2b,c. Each unit cell includes three BPDA molecules, two parallel to each other and perpendicular to the third one. There are thus two nonequivalent directions in an extended domain of the B phase: single-row molecular chains along one direction and double-row molecular chains along the other direction. Similar to the A phase, the basic bonding configuration of the BPDA molecules in the B phase is also a distorted cross, which, however, consists of two "T"-shaped motifs (with one rotated by $180^{\circ}$ ) and includes six BPDA molecules in total, as shown in Figure 2b,c. Force field and DFT calculations unveil that the three molecules inside a " $T$ "shaped structure are connected via two single HBs (blue single dashed lines in Figure $3 b$ ), leading to the formation of a halfcycle of HBs (graph-set notation $\left.D_{2}^{2}(6)\right)$. ${ }^{13}$ Two "T"-shaped structures are bound via weak double HBs between the hydroxyl subgroups of the carboxyls (red double dashed line in Figure $3 b)$. The rotation of the long axes of BPDA molecules away from the lattice vectors of the molecular networks also imprints chirality onto the B phase (Figure $2 b, c$ ), similar to the A phase. Figure $3 b$ shows the calculated models of the $\lambda$ - and $\rho$-chiral domains and the connection of the distorted cross of the $\mathrm{B}$ phase via two different types of HBs.

As the BPDA molecules in the B phase adopt rather similar configurations to those in the A phase, it is not surprising that the $\mathrm{A}$ and $\mathrm{B}$ phases perfectly match without introduction of disorder at domain boundaries. The B phase can also be viewed as an "inflation" of the A phase by addition of one more row (with two molecules) into its cross configuration. Insertion of one more row of BPDA molecules into the distorted cross of the $\mathrm{B}$ phase leads to the formation of the still higher-order structure of the $\mathrm{C}$ phase (Figure 2d,e), which can also seamlessly connect to the $\mathrm{A}$ or $\mathrm{B}$ phases. The structural model for the $\mathrm{C}$ phase is given in Figure $3 \mathrm{c}$. Because the $\mathrm{C}$ phase is structurally more complex than the $\mathrm{A}$ and $\mathrm{B}$ phases, the formation of extended domains of this phase is kinetically hindered, resulting in less frequent observation than the $\mathrm{A}$ and $\mathrm{B}$ phases.

In contrast to the coordination bonding between the deprotonated carboxylic groups and $\mathrm{Fe}$ atoms in the Fe-BPDA metal-organic coordination networks reported by Stepanow and co-workers, ${ }^{6,7}$ in the present case, the formation of $\mathrm{H}$-bonded open networks of BPDA molecules requires no additional deposition of metal atoms onto the $\mathrm{Au}(111)$ surface. Although, at first glance, the observed open networks appear rather similar to the ones formed by the coordination of BPDA molecules and iron atoms on $\mathrm{Cu}(100),{ }^{6,7}$ we are convinced that no $\mathrm{Au}$ adatoms are involved in the formation of the open networks of the A, B, and C phases. High-resolution STM images reveal the absence of additional features corresponding to Au adatoms and different detailed structures from the metal-coordinated ones, consistent with the force field and DFT calculations. Furthermore, if the open networks were Au-coordinated, one would expect an obvious increase of the ratio of open networks to close-packed domains of BPDA molecules after sample annealing to elevated temperatures because annealing would provide more $\mathrm{Au}$ adatoms and activate the deprotonation of the carboxyl groups and thus promote a structural transition from close-packed domains to metal-coordinated open networks. However, we did not observe such an increase after sample annealing up to $\geq 120^{\circ} \mathrm{C}$. Therefore, we conclude that the open networks are stabilized by HBs between carboxyl groups and $\mathrm{vdW}$ interactions rather than $\mathrm{Au}-\mathrm{BPDA}$ coordination. The fully protonated carboxylic groups of BPDA molecules are linked through cross-shaped tetramer (in the A phase) and " $T$ "-shaped half-cyclic trimer (in the $\mathrm{B}$ and $\mathrm{C}$ phases) motifs, which are further bonded via weak double HBs between hydroxyl subgroups. These unique binding arrangements demonstrate the high flexibility of carboxylic acids in supramolecular self-assembly.

We have previously reported the formation of a series of honeycomb networks with increasing interpore distance after the self-assembly of $C_{3}$-symmetric hexaphenylbenzene (HPB) molecules on $\mathrm{Au}(111)$ surfaces and proposed that both the Desiraju-Wuest postulate and Kitaigorodskii's principle of close-packing play important roles in determining 2D molecular crystal structures. ${ }^{16}$ In the present case of $C_{2}$-symmetric BPDA molecules, the $\mathrm{A}, \mathrm{B}$, and $\mathrm{C}$ phases also constitute a series of open networks with increasing interpore distance along one lattice vector and a constant pore-to-pore distance along the other direction. Molecular force field calculations (Figure 4) unveil that the intermolecular interaction energies per molecule of different phases increase in the order of close-packing $<\mathrm{A}<\mathrm{B}$ $<\mathrm{C}$, whereas the intermolecular interaction energies per area of different phases increase in the order of close-packing $<\mathrm{C}<$ $\mathrm{B}<\mathrm{A}$, indicating that the close-packing phase is energetically most stable and the $\mathrm{A}, \mathrm{B}$, and $\mathrm{C}$ phases are metastable with nearly degenerate interaction energies. However, we note that the molecule-substrate interaction is not considered in our force field calculations, except that the molecules are forced to lie within the same plane. It is well-known that the final structure of the molecular self-assembly at surfaces is governed by the subtle balance of intermolecular interaction and moleculesubstrate interaction. The coexistence of the close-packing islands and the open networks of the $\mathrm{A}, \mathrm{B}$, and $\mathrm{C}$ phases suggests that the molecule-substrate interaction should be taken into account and all observed phases are metastable. Unfortu- 


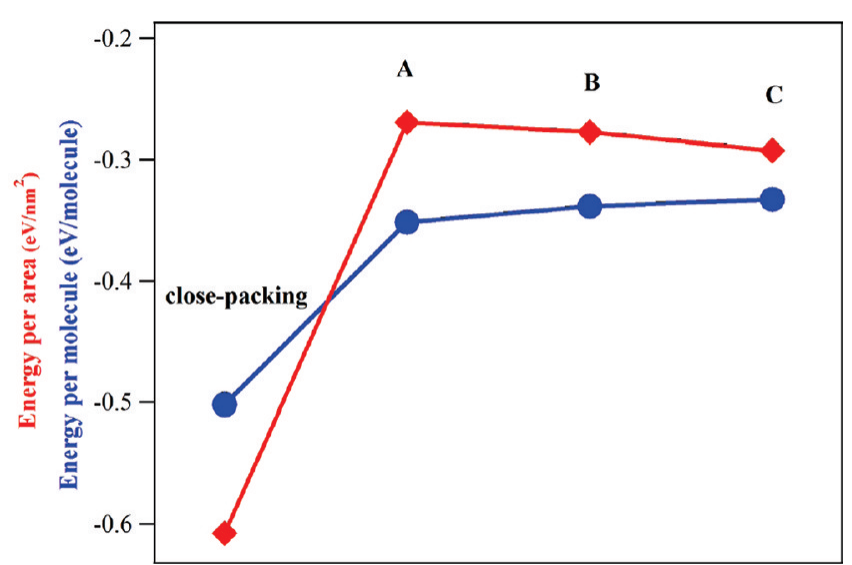

Figure 4. Interaction energies per molecule and interaction energies per area of the close-packing, A, B, and C phases obtained from Amber3 force field calculations.

nately, a precise calculation of the molecule-substrate interaction energy is hindered by the large unit cells of the molecular superstructures and our limited computational capability. Recently, Zhang et al. observed a series of open networks for $C_{3^{-}}$ symmetric 2,4,6-tris(2-pyridyl)-1,3,5-triazine (TPTZ) molecules self-assembling on the $\mathrm{Au}(111)$ surface, but with the absence of certain superstructure orders. ${ }^{17}$ This behavior was rationalized by taking the molecule-substrate interaction into account.

\section{Conclusions}

In summary, we have investigated the self-assembly of BPDA molecules on $\mathrm{Au}(111)$ at the submolecular level by STM. We observe the formation of a variety of $\mathrm{H}$-bonded 2D chiral porous networks as well as close-packed islands. The capability to form various hydrogen-bonding motifs with carboxyl groups highlights the flexibility of carboxylic acids in the synthesis of surface-supported supramolecular architectures.

Acknowledgment. Financial support by the European Commission (RADSAS, NMP3-CT-2004-001561), the Swiss Na- tional Science Foundation, and the National Science Foundation of China is gratefully acknowledged.

Supporting Information Available: Force field and DFT calculations. This material is available free of charge via the Internet at http://pubs.acs.org.

\section{References and Notes}

(1) Barth, J. V. Annu. Rev. Phys. Chem. 2007, 58, 375.

(2) Lackinger, M.; Heckl, W. M. Langmuir 2009, 25, 11307.

(3) Stepanow, S.; Lingenfelder, M.; Dmitriev, A.; Spillmann, H.; Delvigne, E.; Lin, N.; Deng, X.; Cai, C.; Barth, J. V.; Kern, K. Nat. Mater. 2004, 3, 229.

(4) Leiserowitz, L. Acta Crystallogr. 1976, B32, 775.

(5) Stepanow, S.; Lin, N.; Vidal, F.; Landa, A.; Ruben, M.; Barth, J. V.; Kern, K. Nano Lett. 2005, 5, 901.

(6) Stepanow, S.; Lin, N.; Barth, J. V.; Kern, K. J. Phys. Chem. B 2006, 110, 23472.

(7) Lin, N.; Stepanow, S.; Vidal, F.; Barth, J. V.; Kern, K. Chem. Commun. 2005, 1681.

(8) Zhu, N.; Osada, T.; Komeda, T. Surf. Sci. 2007, 601, 1789.

(9) HyperChem Release 7.0; Hypercube Inc.: Gainesville, FL, http:// www.hyper.com/.

(10) Granovsky, A. A. PC GAMESS, version 7.0; http://classic.chem.msu.su/gran/gamess/index.html.

(11) Xu, X.; Goddard, W. A., III Proc. Natl. Acad. Sci. U.S.A. 2004, 101, 2673.

(12) Barth, J. V.; Brune, H.; Ertl, G.; Behm, R. J. Phys. Rev. B 1990, 42, 9307.

(13) Etter, M. C.; MacDonald, J. C.; Bernstein, J. Acta Crystallogr. 1990, $B 46,256$.

(14) (a) Cañas-Ventura, M. E.; Klappenberger, F.; Clair, S.; Pons, S.; Kern, K.; Brune, H.; Strunskus, T.; Wrill, Ch.; Fasel, R.; Barth, J. V. J. Chem. Phys. 2006, 125, 184710. (b) Heininger, C.; Kampschulte, L.; Heckl, W. M.; Lackinger, M. Langmuir 2009, 25, 968.

(15) Eichhorst-Gerner, K.; Stabel, A.; Moessner, G.; Declerq, D.; Valiyaveettil, S.; Enkelmann, V.; Müllen, K.; Rabe, J. P. Angew. Chem., Int. Ed. 1996, 35, 1492.

(16) Xiao, W.; Feng, X.; Ruffieux, P.; Gröning, O.; Müllen, K.; Fasel, R. J. Am. Chem. Soc. 2008, 130, 8910.

(17) Zhang, J.; Li, B.; Cui, X.; Wang, B.; Yang, J.; Hou, J. G. J. Am. Chem. Soc. 2009, 131, 5885.

JP100701Y 